\title{
DETERMINAÇÃo SIMULTÂNEA dE As, Cd E Pb EM AMOSTRAS DE ÁGUA PURIFICADA PARA HEMO- DIÁLISE POR ESPECTROMETRIA DE ABSORÇÃO ATÔMICA COM FORNO DE GRAFITE, APÓS OTIMI- ZAÇÃO MULTIVARIADA BASEADA NO USO DE PLANEJAMENTO EXPERIMENTAL
}

Lisia Maria G. dos Santos*, Jaylei M. Gonçalves e Silvana do Couto Jacob

Departamento de Química, Instituto Nacional de Controle de Qualidade em Saúde, Fundação Oswaldo Cruz, Av. Brasil, 4365, 21040-360 Rio de Janeiro - RJ, Brasil

Recebido em 19/12/06; aceito em 30/11/07; publicado na web em 24/7/08

SIMULTANEOUS DETERMINATION OF ARSENIC, CADMIUM AND LEAD BY GF AAS IN PURIFIED WATER SAMPLES FOR HEMODIALYSIS AFTER MULTIVARIATE OPTIMIZATION BASED ON FACTORIAL DESIGN. This paper reports the development of a methodology for simultaneously determining $\mathrm{As}, \mathrm{Cd}$ and $\mathrm{Pb}$, employing GF AAS with polarized Zeeman-effect background correction. In order to make the procedure applicable, the influence of pyrolysis and atomization temperatures and the amount of chemical modifiers were studied. Factorial and central composite designs were used to optimize these variables. Precision and accuracy of the method were investigated using Natural Water Reference material, Nist SRM 1640. Results are in agreement with certified values at the $95 \%$ confidence limit when the Student $t$-test is used. This methodology was used for quality control of purified water for hemodialysis.

Keywords: factorial design; purified water for hemodialysis; inorganic contamination.

\section{INTRODUÇÃO}

A contaminação das águas por substâncias químicas tóxicas é uma ameaça à qualidade da vida humana e, de modo especial, para aquelas pessoas que sofrem de insuficiência renal e necessitam de hemodiálise.

A hemodiálise é um processo de filtragem e depuração das substâncias indesejáveis do sangue, como a creatinina e a uréia, realizada em pacientes portadores de insuficiência renal crônica ou aguda. No processo de hemodiálise, o sangue do paciente é retirado e impulsionado, por uma bomba, até o filtro de diálise, conhecido como dialisador. No dialisador, o sangue é então exposto à solução de diálise $(\mathrm{Na}, \mathrm{K}$, bicarbonato, $\mathrm{Ca}, \mathrm{Mg}, \mathrm{Cl}$, acetato) através de uma membrana semipermeável, permitindo as trocas entre as substâncias do sangue e do dialisato. Posteriormente, o sangue "filtrado" é devolvido ao paciente pelo acesso vascular.

A água utilizada no processo de hemodiálise deve obedecer à Resolução RDC n ${ }^{\circ}$ 154, de 15 de Junho de 2004, ${ }^{1}$ da Agência Nacional de Saúde (ANVISA). Esta resolução estabelece os limites máximos permitidos para contaminantes inorgânicos na água para diálise, sendo que arsênio, cádmio e chumbo estão entre os principais contaminantes a serem controlados. Estes contaminantes, por serem extremamente tóxicos, fazem parte do programa de controle da qualidade da água para diálise, realizado pelo Instituto Nacional de Controle de Qualidade em Saúde (INCQS/FIOCRUZ), a fim de monitorar e garantir a segurança deste tratamento.

A concentração dos contaminantes em água ocorre, geralmente, em níveis de traço, sendo necessário, para detectá-los e quantificá-los, o uso de técnicas analíticas de alta sensibilidade, como a espectrometria de absorção atômica com forno de grafite (GF AAS), ${ }^{2,3}$ utilizada neste trabalho.

Devido aos fatores mencionados, houve a necessidade do desenvolvimento de uma metodologia para a determinação, na rotina, das concentrações dos elementos de interesse (arsênio, cádmio e chum-

*e-mail: lisia.maria@rjnet.com.br bo) de forma simultânea, minimizando custos e aumentando a rapidez da resposta analítica, de modo a permitir maior controle do tratamento por parte dos hemocentros. Para tanto, foi empregado um planejamento fatorial experimental, a fim de otimizar a rotina da determinação de arsênico, cádmio e chumbo por GF AAS. O planejamento fatorial experimental é uma ferramenta estatística que, por sua simplicidade, vem sendo cada vez mais utilizada pelos químicos analíticos, pois permite avaliar as condições ideais de trabalho e a otimização de metodologias. ${ }^{4}$ Em especial, utilizando as técnicas espectroanalíticas, podemos citar Cassella et al., ${ }^{5}$ que empregaram planejamento fatorial de segunda ordem para obter as condições ideais na determinação de arsênio em amostras de petróleo, por GF AAS. Outro trabalho citado na literatura é o de Pereira-Filho et al., ${ }^{6}$ onde é apresentada uma metodologia para obtenção dos valores ótimos para as temperaturas de pirólise e atomização na determinação de $\mathrm{Al}, \mathrm{Cd}, \mathrm{Mo}$ e $\mathrm{Pb}$, usando planejamento fatorial.

Para executar um planejamento fatorial experimental é preciso determinar quais os fatores (variáveis independentes) e qual a resposta de interesse (variável dependente) para o sistema que se deseja estudar. Também é preciso definir que objetivo se quer alcançar com os experimentos, porque isto definirá que tipo de planejamento deverá ser utilizado. Devido a sua simplicidade e baixo custo, o planejamento fatorial de dois níveis, completo ou fracionário, ${ }^{4}$ é muito utilizado em estudos preliminares ou como passo inicial para uma otimização. Neste tipo de planejamento, cada fator é estudado em dois níveis (máximo e mínimo) e, portanto, supõe-se uma linearidade nos efeitos dos fatores. Para a obtenção de uma superfície de resposta não linear, e obtenção de um ponto ótimo, é necessário avaliação de um maior número de pontos. ${ }^{7,8}$

Neste trabalho foi feito, inicialmente, um planejamento fatorial fracionário de dois níveis $\left(2^{4-1}\right)$, com um ponto central, para avaliar os fatores que apresentavam maiores efeitos na resposta analítica do método. Posteriormente, com os resultados obtidos, procurou-se um ponto ótimo para as temperaturas de pirólise e atomização que satisfizesse as condições ideais para determinar, simultaneamente, As, $\mathrm{Cd}$ e $\mathrm{Pb}$ através da metodologia de superfície de resposta (RSM). 


\section{PARTE EXPERIMENTAL}

\section{Instrumentação}

Todos os experimentos foram realizados num espectrômetro de absorção atômica com forno de grafite e detecção simultânea de até quatro elementos (SIMAA 6000, da Perkin Elmer). O equipamento é dotado de tubo de grafite, com aquecimento transversal e plataforma integrada recoberta com grafite pirolítico, corretor de fundo tipo efeito Zeeman, amostrador automático (As-72) e lâmpadas de descarga, sem eletrodos (EDL), de arsênio, cádmio e chumbo. ${ }^{9,10}$

Durante o estudo das variáveis, mantiveram-se fixos os seguintes parâmetros: volume de injeção das amostras e dos padrões em $20 \mu \mathrm{L}$, volume dos modificadores em $5 \mu \mathrm{L}$ e temperaturas de secagem em 110 e $130{ }^{\circ} \mathrm{C}$.

\section{Reagentes, soluções e amostras}

Todas as soluções foram preparadas usando água ultrapura, desionizada em sistema de purificação Milli-Q (Millipore). Os reagentes foram adquiridos da empresa Merck e utilizados dentro dos respectivos prazos de validade.

Solução analítica contendo $4 \mu \mathrm{g} \mathrm{L}^{-1}$ de arsênio, $1 \mu \mathrm{g} \mathrm{L}^{-1}$ de cádmio, $20 \mu \mathrm{g} \mathrm{L}^{-1}$ de chumbo e $0,2 \% \mathrm{v} / \mathrm{v}$ de $\mathrm{HNO}_{3}$, usado como conservante. Esta solução foi preparada, diariamente, pela diluição da solução estoque, contendo $1000 \mathrm{mg} \mathrm{L}^{-1}$ (Tritisol Merck) de As $\left(\mathrm{H}_{3} \mathrm{AsO}_{4}\right), \mathrm{Cd}\left(\mathrm{CdCl}_{2}\right)$ e $\mathrm{Pb}\left[\mathrm{Pb}\left(\mathrm{NO}_{3}\right)_{2}\right]$. Uma solução com $0,2 \%$ $\mathrm{v} / \mathrm{v}$, na ausência destes elementos, foi preparada e utilizada como branco. Os modificadores químicos foram preparados com sais de elevado grau de pureza destinados a esta finalidade, contendo $10.000 \mathrm{mg} \mathrm{L}^{-1}$ de $\mathrm{Pd}\left(\mathrm{NO}_{3}\right)_{2}$ e $\mathrm{Mg}\left(\mathrm{NO}_{3}\right)_{2}$ (Suprapur, Merck).

Para controle da qualidade dos resultados analíticos, utilizaram-se amostras de referência de água certificadas pelo National Institute of Standards and Technology (Nist, Natural Water $\mathrm{n}^{\circ} 1640$ ), com teores conhecidos de As $\left(26,67 \pm 0,41 \mu \mathrm{g} \mathrm{kg}^{-1}\right), \mathrm{Cd}(22,79 \pm$ $\left.0,96 \mu \mathrm{g} \mathrm{kg}^{-1}\right)$ e $\mathrm{Pb}\left(27,89 \pm 0,14 \mu \mathrm{g} \mathrm{kg}^{-1}\right)$.

As amostras para análise foram coletadas em hemocentros da cidade do Rio de Janeiro, pela Secretaria Estadual de Saúde, e encaminhadas ao INCQS/FIOCRUZ. As coletas foram efetuadas em diferentes pontos do processo de tratamento da água: entrada da rede, osmose e pós-osmose reversa.

\section{RESULTADOS E DISCUSSÃO}

Estudo das variáveis independentes: temperatura de pirólise, temperatura de atomização e modificadores químicos

Para desenvolver o processo de análise simultânea de As, Cd e $\mathrm{Pb}$, por GF AAS, primeiramente, foi proposto um planejamento fatorial fracionário de dois níveis, com ponto central, para verificar quais variáveis, dentre as previamente selecionadas (temperatura de atomização, temperatura de pirólise e concentração dos modificadores químicos) poderiam causar maior variação na resposta analítica.

Os níveis adotados para as variáveis de temperatura basearamse no comportamento eletrotérmico dos elementos e nas temperaturas utilizadas nas determinações mono elementar de $\mathrm{As}, \mathrm{Cd}$ e $\mathrm{Pb}$. Em geral, a temperatura de pirólise é selecionada pelo elemento mais volátil e a temperatura de atomização pelo elemento menos volátil. A escolha dos modificadores químicos baseou-se na literatura disponível, onde se verificou que, apesar de haver diversas substâncias sendo usadas como modificadores químicos, a mistura
$\mathrm{Pd}+\mathrm{Mg}\left(\mathrm{NO}_{3}\right)_{2}$ é conhecida, desde o começo dos anos 90, como o "modificador universal" por apresentar bom desempenho para a grande maioria dos elementos determinados por GF AAS. ${ }^{3,11}$ Em função disso, optamos por trabalhar com estes dois modificadores, sendo as concentrações adotadas baseadas nas determinações mono elementares dos três elementos ( $\mathrm{As}, \mathrm{Cd}, \mathrm{Pb})$.

Um planejamento fatorial completo, no estudo proposto, requereria 16 experimentos ( $2^{4}$ - quatro fatores), mas, como o objetivo era selecionar os fatores que mais influenciariam na resposta analítica, optou-se por um planejamento fracionário $2^{4-1}$, onde apenas 8 experimentos são necessários. Este tipo de planejamento despreza os termos de ordem superior que, geralmente, são os menos significativos. ${ }^{7}$ Todos os fatores foram analisados em dois níveis (máximo e mínimo) e num ponto central, realizado em quadruplicata, totalizando 12 experimentos. Na Tabela 1 encontramos os níveis das temperaturas e dos modificadores que foram estudados.

Tabela 1. Fatores e níveis do planejamento fatorial $2^{4-1}$ com ponto central

\begin{tabular}{lccc}
\hline Fatores & $\begin{array}{c}\text { Mínimo } \\
(-1)\end{array}$ & $\begin{array}{c}\text { Ponto central } \\
(0)\end{array}$ & $\begin{array}{c}\text { Máximo } \\
(+1)\end{array}$ \\
\hline $\begin{array}{l}\text { Temperatura de } \\
\text { pirólise }\left({ }^{\circ} \mathrm{C}\right)\end{array}$ & 600 & 800 & 1000 \\
$\begin{array}{l}\text { Temperatura de } \\
\text { atomização }\left({ }^{\circ} \mathrm{C}\right)\end{array}$ & 1400 & 1800 & 2200 \\
$\begin{array}{l}\text { Concentração de } \\
\text { modificador }\end{array}$ & 0,6 & 1,2 & 1,8 \\
$\begin{array}{l}\text { Mg }\left(\mathrm{NO}_{3}\right)_{2}-\left(\mathrm{mg} \mathrm{L}^{-1}\right) \\
\text { Concentração de } \\
\text { modificador }\end{array}$ & 0,5 & 1 & 2 \\
Pd $\left(\mathrm{NO}_{3}\right)_{2}-\left(\mathrm{mg} \mathrm{L}^{-1}\right)$ & & & \\
\hline
\end{tabular}

A execução do planejamento consistiu em realizar os ensaios e registrar as respostas analíticas observadas (absorvâncias), para todas as combinações de níveis.

As respostas analíticas obtidas, através dos resultados experimentais, foram inseridas no programa Statistica $6.0,{ }^{12}$ o que permitiu avaliar as interações entre as variáveis estudadas e a sua influência sobre a resposta analítica de interesse.

Este planejamento fracionário foi utilizado para detectar a magnitude dos efeitos das variáveis independentes sobre as variáveis dependentes. Isto foi verificado pela aplicação da análise de variância (ANOVA) e pela estimativa do erro experimental (erro puro), que permite verificar de maneira quantitativa se o modelo representado é satisfatório, o que só é possível através das replicatas do ponto central, uma vez que não se fez replicatas dos demais pontos. Conforme se pode observar nas Figuras 1, 2 e 3 os fatores

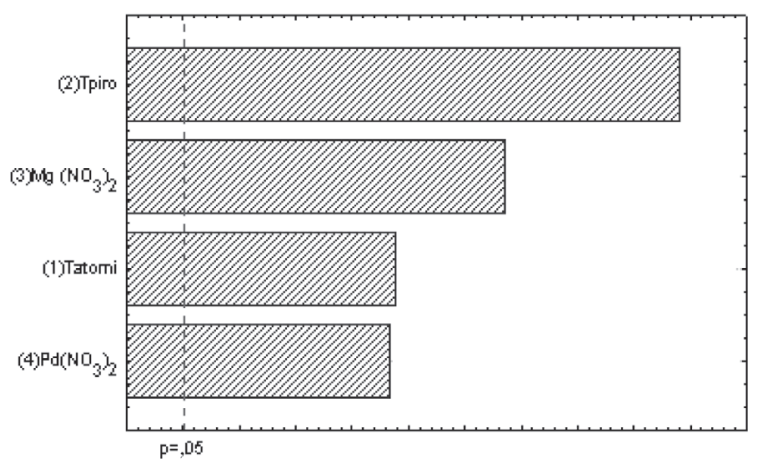

Figura 1. Gráfico de Pareto mostra os efeitos das variáveis sobre a sensibilidade da metodologia para o arsênio 


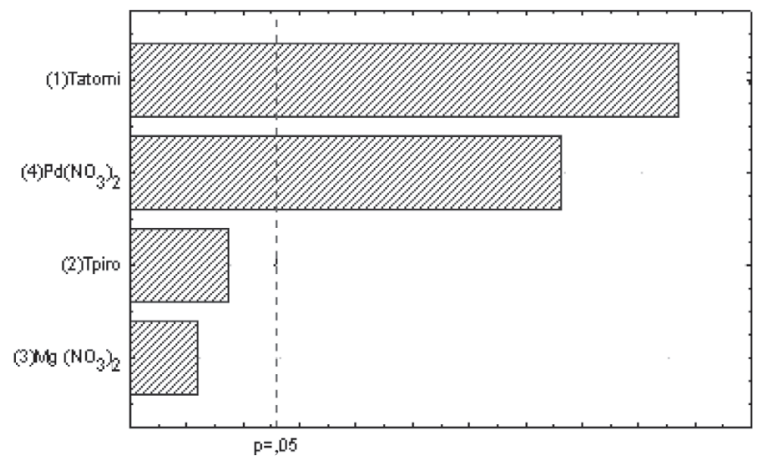

Figura 2. Gráfico de Pareto mostra os efeitos das variáveis sobre a sensibilidade da metodologia para cádmio

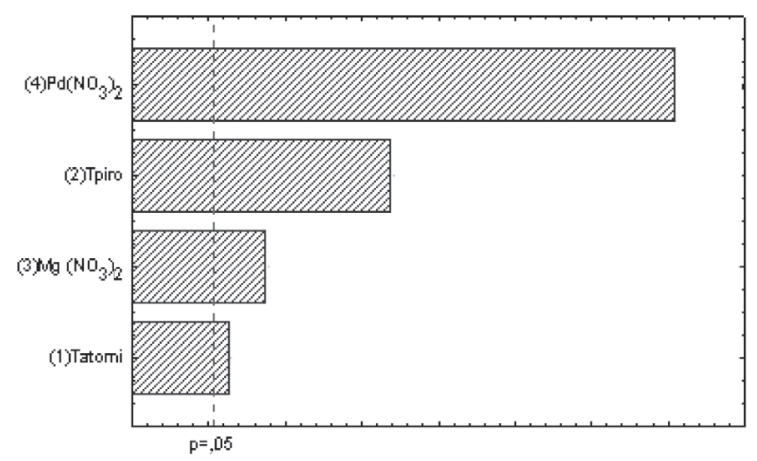

Figura 3. Gráfico de Pareto mostra os efeitos das variáveis sobre a sensibilidade da metodologia para o chumbo

que tiveram um p-level menor que 0,05 são, estatisticamente, significativos e podem influenciar de maneira positiva ou negativa no resultado esperado.

\section{Otimização multivariada}

Após a realização dos experimentos de triagem, os fatores significativos foram selecionados e a metodologia de análise de superfície de resposta foi executada para obtenção do ponto ótimo. Esta metodologia baseia-se na construção de modelos matemáticos empíricos que geralmente empregam funções polinomiais, quadráticas ou de ordem superior, para descreverem o sistema estudado e, conseqüentemente, dão condições de explorar (modelar e deslocar) o sistema até atingir uma região ótima (máximo e mínimo) da superfície de resposta investigada. ${ }^{7,8}$

O processo de otimização para determinação simultânea de arsênio, cádmio e chumbo por GF AAS foi dividido em duas etapas: primeiro foi realizada a otimização da temperatura de pirólise e do modificador $\mathrm{Pd}\left(\mathrm{NO}_{3}\right)_{2}$ e, posteriormente, a otimização das temperaturas de pirólise e de atomização. Este método permitiu chegar ao valor ótimo da resposta através de um pequeno número de experimentos.

\section{Planejamento 1}

Nessa etapa, utilizou-se a metodologia de superfície de resposta em torno das condições previamente estabelecidas: temperatura de pirólise, de 600 a $1000{ }^{\circ} \mathrm{C}$, e concentração do modificador químico, de 0,5 a $2 \mathrm{mg} \mathrm{L}^{-1}$.

Para atingir a região ótima foi feito um planejamento fatorial de dois níveis com pontos centrais e axiais (Tabela 2).

Neste planejamento, a temperatura de atomização $\left(2000{ }^{\circ} \mathrm{C}\right)$ e
Tabela 2. Planejamento 1: Valores para a temperatura de pirólise e concentração de $\mathrm{Pd}\left(\mathrm{NO}_{3}\right)_{2}$ usados no planejamento composto central; temperatura de atomização $=2000{ }^{\circ} \mathrm{C}$; concentração do modificador $\mathrm{Mg}\left(\mathrm{NO}_{3}\right)_{2}=0,6 \mathrm{mg} \mathrm{L}^{-1}$

\begin{tabular}{lccccc}
\hline & $\begin{array}{c}\mathrm{T} \\
\text { pirólise } \\
\left({ }^{\circ} \mathrm{C}\right)\end{array}$ & $\begin{array}{c}\mathrm{Pd}\left(\mathrm{NO}_{3}\right)_{2} \\
\mathrm{mg} \mathrm{L}^{-1}\end{array}$ & $\begin{array}{c}\text { Abs } \\
\text { Integrada } \\
\text { As }\end{array}$ & $\begin{array}{c}\text { Abs } \\
\text { Integrada } \\
\mathrm{Cd}\end{array}$ & $\begin{array}{c}\text { Abs } \\
\text { Integrada } \\
\mathrm{Pb}\end{array}$ \\
\hline 1 & 600 & 0,500 & 0,0026 & 0,0126 & 0,0122 \\
2 & 600 & 2,000 & 0,0051 & 0,0107 & 0,0122 \\
3 & 1000 & 0,500 & 0,0032 & 0,0026 & 0,0142 \\
4 & 1000 & 2,000 & 0,0020 & 0,0059 & 0,0096 \\
5 & 517 & 1,000 & 0,0025 & 0,0130 & 0,0118 \\
6 & 1083 & 1,000 & 0,0044 & 0,0110 & 0,0890 \\
7 & 800 & $\mathrm{Zero}$ & 0,0050 & 0,0052 & 0,0137 \\
8 & 800 & 2,414 & 0,0042 & 0,0086 & 0,0112 \\
$9(\mathrm{C})$ & 800 & 1,000 & 0,0055 & 0,0081 & 0,0107 \\
$10(\mathrm{C})$ & 800 & 1,000 & 0,0043 & 0,0097 & 0,0120 \\
$11(\mathrm{C})$ & 800 & 1,000 & 0,0059 & 0,0085 & 0,0110 \\
12 (C) & 800 & 1,000 & 0,0045 & 0,0086 & 0,0117 \\
13 (C) & 800 & 1,000 & 0,00540 & 0,00950 & 0,01210 \\
\hline
\end{tabular}

a concentração do modificador $\mathrm{Mg}\left(\mathrm{NO}_{3}\right)_{2}\left(0,6 \mathrm{mg} \mathrm{L}^{-1}\right)$ foram mantidas constantes.

Com dados da Tabela 2 e através da opção Central composite, non-factorial, surface design do programa Statistica 6.0, ${ }^{12}$ construiu-se uma superfície de resposta, representando a interação entre a temperatura de pirólise e a concentração do modificador $\mathrm{Pd}\left(\mathrm{NO}_{3}\right)_{2}$. Este planejamento permitiu verificar a presença de um ponto crítico, através da aplicação do critério de Lagrange, levando à obtenção das coordenadas do ponto máximo da superfície. Este ponto representa a situação onde se obtém a maior sensibilidade para o método. A Tabela 3 apresenta estes valores para os elementos em estudo.

Tabela 3. Valores ótimos obtidos, segundo o planejamento 1

\begin{tabular}{lccr}
\hline & Arsênio & Cádmio & Chumbo \\
\hline Temperatura de pirólise & $800{ }^{\circ} \mathrm{C}$ & $900{ }^{\circ} \mathrm{C}$ & $700{ }^{\circ} \mathrm{C}$ \\
Concentração $\mathrm{Pd}\left(\mathrm{NO}_{3}\right)_{2}$ & $1,0 \mathrm{mg} \mathrm{L}^{-1}$ & $1,5 \mathrm{mg} \mathrm{L}^{-1}$ & $0,9 \mathrm{mg} \mathrm{L}^{-1}$ \\
\hline
\end{tabular}

No entanto, neste estudo, busca-se encontrar os valores operacionais ótimos das variáveis independentes que satisfaçam, simultaneamente, todas as variáveis dependentes e que levem a maior sensibilidade. Para tal usou-se a otimização com restrições, que recebe o nome de método de programação não linear. ${ }^{7} \mathrm{O}$ Statistica 6.0 usa a abordagem usada por Derringer e Suich (1980), que utiliza as funções desirability. Com esta abordagem foi possível encontrar os valores operacionais ótimos das variáveis independentes que satisfazem, simultaneamente, as variáveis dependentes.

Os valores encontrados para estas variáveis foram: temperatura de pirólise $=1000{ }^{\circ} \mathrm{C}$ e concentração do modificador $\mathrm{Pd}\left(\mathrm{NO}_{3}\right)_{2}=$ $1 \mathrm{mg} \mathrm{L}^{-1}$. Estes valores correspondem ao ponto de maior absorvância e, portanto, ao ponto de maior sensibilidade para os três elementos, arsênio, cádmio e chumbo.

Analisando estes resultados, percebemos que a temperatura de pirólise, para o elemento cádmio, foi muito alta (Figura 4), comparada com a temperatura utilizada na análise mono elementar.

Em função disso, optou-se pela realização de um segundo planejamento, onde avaliamos a temperatura de atomização, por ser a variável de maior influência na análise do cádmio, conforme mos- 


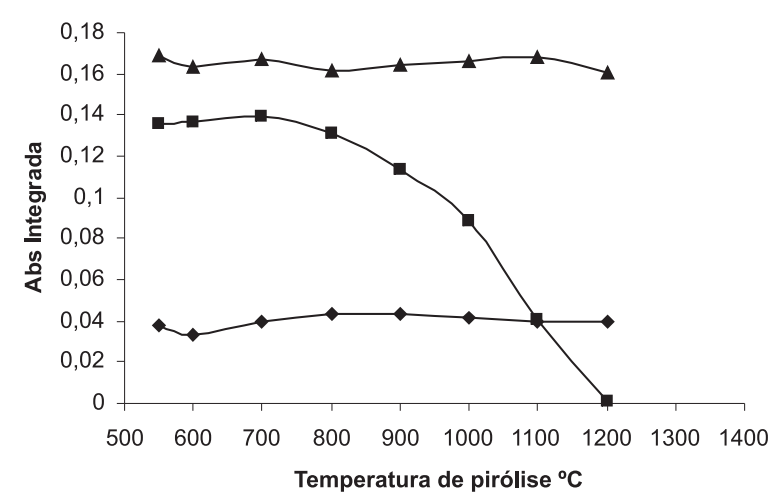

Figura 4. Curva de pirólise para os diferentes elementos em estudo com 0,6 $m g \mathrm{~L}^{-1}$ de $\mathrm{Mg}\left(\mathrm{NO}_{3}\right)_{2}$ e temperatura de atomização $2000^{\circ} \mathrm{C}:(\bullet)$ arsênio, (•) cádmio e ( $\mathbf{\Delta})$ chumbo

trado no estudo preliminar, junto com a temperatura de pirólise, que representa o ponto crítico para análise desse elemento.

\section{Planejamento 2}

Neste planejamento, a concentração do modificador $\mathrm{Pd}\left(\mathrm{NO}_{3}\right)_{2}$ foi mantida igual a $1 \mathrm{mg} \mathrm{L}^{-1}$, segundo a otimização anterior, e a concentração do modificador $\mathrm{Mg}\left(\mathrm{NO}_{3}\right)_{2}$ igual a $0,6 \mathrm{mg} \mathrm{L}^{-1}$, porque esta variável não tem um efeito significativo na sensibilidade do método para cádmio e chumbo. Optou-se por adotar a concentração usada para a análise mono elementar do elemento arsênio, visto que este elemento sofre maior influência desta variável nos resultados.

Os experimentos realizados estão indicados na Tabela 4. Estes resultados foram manipulados seguindo o mesmo caminho do planejamento 1 .

Tabela 4. Planejamento 2: Valores para as temperaturas de pirólise e de atomização usados no planejamento composto central; concentração de $\mathrm{Pd}\left(\mathrm{NO}_{3}\right)_{2}=1 \mathrm{mg} \mathrm{L}^{-1}$; concentração de $\mathrm{Mg}\left(\mathrm{NO}_{3}\right)_{2}=$ $0,6 \mathrm{mg} \mathrm{L}^{-1}$

\begin{tabular}{lccccc}
\hline & $\begin{array}{c}\mathrm{T} \\
\text { atomização } \\
\left({ }^{\circ} \mathrm{C}\right)\end{array}$ & $\begin{array}{c}\mathrm{T} \\
\text { pirólise } \\
\left({ }^{\circ} \mathrm{C}\right)\end{array}$ & $\begin{array}{c}\text { Abs } \\
\text { Integrada } \\
\text { As }\end{array}$ & $\begin{array}{c}\text { Abs } \\
\text { Integrada } \\
\text { Cd }\end{array}$ & $\begin{array}{c}\text { Abs } \\
\text { Integrada } \\
\text { Pb }\end{array}$ \\
\hline 1 & 1400 & 600 & 0,0037 & 0,0316 & 0,0024 \\
2 & 1400 & 1000 & 0,0002 & 0,0204 & 0,0052 \\
3 & 2200 & 600 & 0,0755 & 0,0260 & 0,1549 \\
4 & 2200 & 1000 & 0,0784 & 0,0176 & 0,1535 \\
5 & 1234 & 800 & 0,0015 & 0,0160 & 0,0002 \\
6 & 2366 & 800 & 0,0739 & 0,0627 & 0,1381 \\
7 & 1800 & 517 & 0,0169 & 0,0261 & 0,1807 \\
8 & 1800 & 1083 & 0,0243 & 0,0035 & 0,1738 \\
$9(\mathrm{C})$ & 1800 & 800 & 0,0175 & 0,0237 & 0,1819 \\
$10(\mathrm{C})$ & 1800 & 800 & 0,0228 & 0,0250 & 0,1842 \\
$11(\mathrm{C})$ & 1800 & 800 & 0,0232 & 0,0251 & 0,1837 \\
12 (C) & 1800 & 800 & 0,0220 & 0,0245 & 0,1839 \\
13 (C) & 1800 & 800 & 0,0225 & 0,0253 & 0,1840 \\
\hline
\end{tabular}

Os valores encontrados para as variáveis foram: temperatura de pirólise $=800{ }^{\circ} \mathrm{C}$ e temperatura de atomização $=2200{ }^{\circ} \mathrm{C}$, que correspondem ao ponto de maior sensibilidade para os três elementos, arsênio, cádmio e chumbo. A Tabela 5 apresenta o programa de temperaturas que apresentou as melhores condições para atingir o objetivo deste trabalho.
Tabela 5. Programa de temperaturas que levou à melhor resposta

\begin{tabular}{lcccccc}
\hline Fases & $\begin{array}{c}\text { Temperatura } \\
\left({ }^{\circ} \mathrm{C}\right)\end{array}$ & $\begin{array}{c}\text { Rampa } \\
(\mathrm{s})\end{array}$ & $\begin{array}{c}\text { Tempo } \\
(\mathrm{s})\end{array}$ & $\begin{array}{c}\text { Fluxo } \\
\text { Interno } \\
\left(\mathrm{mL} \mathrm{min}^{-1}\right)\end{array}$ & Gás Leitura \\
\hline 1 & 110 & 1 & 30 & 250 & $\mathrm{~N}$ & \\
2 & 130 & 15 & 30 & 250 & $\mathrm{~N}$ & \\
3 & 800 & 10 & 20 & 250 & $\mathrm{~N}$ & \\
4 & 2200 & 0 & 3 & 250 & - & $*$ \\
5 & 2450 & 1 & 3 & 250 & $\mathrm{~N}$ & \\
\hline
\end{tabular}

\section{Análise das características do método}

Com a definição das condições ideais para a metodologia proposta, realizamos o estudo dos parâmetros de validação da mesma.

A Tabela 6 apresenta as figuras de mérito da metodologia estudada para análise dos elementos de interesse.

Tabela 6. Figuras de mérito para análise simultânea de arsênio, cádmio e chumbo por GF AAS - modelo SIMAA 6000

\begin{tabular}{lccc}
\hline Parâmetros & Arsênio & Cádmio & Chumbo \\
\hline Faixa de Trabalho $\left(\mu \mathrm{g} \mathrm{L}^{-1}\right)$ & $4-20$ & $1-5$ & $20-100$ \\
$\%$ RSD & 1,8 & 1,3 & 0,9 \\
$\mathrm{LD}\left(\mu \mathrm{g} \mathrm{L}^{-1}\right)$ & 1,0 & 0,1 & 1,0 \\
$\mathrm{LQ}\left(\mu \mathrm{g} \mathrm{L}^{-1}\right)$ & 4,0 & 0,4 & 3,5 \\
\hline
\end{tabular}

A validação da metodologia, segundo os parâmetros do INMETRO, ${ }^{13}$ mostrou que o método estudado apresenta precisão e exatidão, tendo sido obtida uma recuperação que variou entre 98 a $110 \%$, para o arsênio, 97 a 102\%, para o cádmio e 92 a 118\%, para o chumbo.

\section{CONCLUSÃO}

A utilização do planejamento fatorial possibilitou definir quais parâmetros, dentre os selecionados, afetariam, de forma mais significativa, a análise simultânea de arsênio, cádmio e chumbo. Além disso, permitiu analisar o sistema de maneira multivariada, buscando estudar todas as variáveis que compunham o sistema experimental, pois, conforme observado experimentalmente, existe interação entre os parâmetros selecionados, o que não seria possível ser percebido na otimização univariada. O planejamento experimental permitiu eficiência e economia no processo experimental e o uso de métodos estatísticos deu mais objetividade científica nas conclusões

A otimização das variáveis, através do uso da metodologia de superfície de resposta, foi bem sucedida, visto que se obteve as condições ideais para determinação simultânea dos três elementos, mesmo tendo, estes elementos, características diferentes entre si, principalmente em relação à temperatura de volatilização. Pôde-se constatar que o uso desta metodologia, com sensibilidade e exatidão adequadas, possibilitou a redução dos custos com reagentes e equipamentos, levando também a uma maior rapidez na resposta analítica.

$\mathrm{O}$ uso desta ferramenta permitiu a implantação e validação de uma metodologia de Controle de Qualidade da água purificada utilizada nos hemocentros, proporcionando maior rapidez e eficácia para as ações de Vigilância Sanitária, portanto espera-se que o presente estudo sirva para incentivar o uso do planejamento experimental fatorial como uma ferramenta no desenvolvimento de novas metodologias em química analítica. 


\section{AGRADECIMENTOS}

Ao INCQS/FIOCRUZ e à Capes pelo suporte financeiro.

\section{REFERÊNCIAS}

1. Brasil, Portaria $n^{\circ} 154,15$ de Junho de 2004. Estabelece o Regulamento Técnico para o funcionamento dos Serviços de Diálise. Diário Oficial [da] República Federativa do Brasil, Poder Executivo, Brasília, DF, 17 jun. 2004.

2. Welz, B.; Sperling, M.; Atomic Absorption Spectrometry - $3^{\text {rd }}$ ed., Completely Revised Edition, Wiley - VCH; Weinheim, 1999.

3. Welz, B.; Schelemmer, G.; Mudakavi, J. R.; J. Anal. At. Spectrom. 1992 7/8, 1257.

4. Costa, M. L.; Korn, M. G. A.; Castro, J. T.; Santos, W. P. C.; Carvalho, E. V.; Nogueira, A. R.A.; Quim. Nova 2006, 29, 149.
5. Cassella, R. J.; Sant'Ana, O. D.; Santelli, R. E.; Spectrochim. Acta, Part B 2002, 57, 1967.

6. Pereira -Fillho, E. R.; Poppi, R. J.; Arruda, M. A. Z.; Quim. Nova 2002, 25,246 .

7. Neto, B. B.; Scarminio, I. S.; Bruns, R. E.; Planejamento e Otimização de Experimentos, Ed. Unicamp: São Paulo, 1996.

8. Calado, V.; Montgomery, D.; Planejamento de Experimento usando o Statistica, Ed.- e- papers: Rio de Janeiro, 2003.

9. Perkin Elmer, The THGA Graphite Furnace: Techniques and Recommended Conditions, 1991.

10. Funk,W.; Dammann, V.; Donnevert, G.; Quality Assurance in Analytical Chemistry, Weinheim-VCH: New York, 1995.

11. Acar, O.; Talanta 2001, 51, 1235.

12. Statistica for Windows; StatSoft, Inc, Tulsa, USA,1999.

13. INMETRO; DOQ-CGCRE-008 -revisão 00: orientação sobre validação de métodos de ensaios químicos, Outubro 2002, http://www.inmetro.gov.br, acessada em Dezembro 2006. 